\title{
Construction of non-constant lower and upper functions for impulsive periodic problems
}

\author{
Irena Rachůnková* and Milan Tvrdý ${ }^{\dagger}$
}

Summary. We present conditions ensuring the existence of piecewise linear lower and upper functions for the nonlinear impulsive periodic boundary value problem $u^{\prime \prime}=f\left(t, u, u^{\prime}\right), u\left(t_{i}+\right)=$ $\mathrm{J}_{i}\left(u\left(t_{i}\right)\right), u^{\prime}\left(t_{i}+\right)=\mathrm{M}_{i}\left(u^{\prime}\left(t_{i}\right)\right), i=1,2, \ldots, m, u(0)=u(T), u^{\prime}(0)=u^{\prime}(T)$. This together with the existence principles which we proved in [5]-[7] allows us to prove new existence criteria, see Theorems 3.1 and 3.2.

Mathematics Subject Classification 2000. 34B37, 34B15, 34C25

Keywords. Second order nonlinear ordinary differential equation, impulse, periodic solution, lower function, upper function.

\section{Introduction}

This paper deals with the impulsive periodic boundary value problem

$$
\begin{gathered}
u^{\prime \prime}=f\left(t, u, u^{\prime}\right), \\
u\left(t_{i}+\right)=\mathrm{J}_{i}\left(u\left(t_{i}\right)\right), \quad u^{\prime}\left(t_{i}+\right)=\mathrm{M}_{i}\left(u^{\prime}\left(t_{i}\right)\right), \quad i=1,2, \ldots, m, \\
u(0)=u(T), \quad u^{\prime}(0)=u^{\prime}(T),
\end{gathered}
$$

where

$$
\left\{\begin{array}{l}
0<t_{1}<\cdots<t_{m}<T<\infty, \\
f \text { satisfies the Carathéodory conditions on }[0, T] \times \mathbb{R}^{2}, \\
\mathrm{~J}_{i} \text { and } \mathrm{M}_{i}, i=1,2, \ldots, m, \text { are continuous functions on } \mathbb{R} .
\end{array}\right.
$$

There are several papers providing the existence results for such problems in terms of lower and upper functions, see e.g. [1]-[4], [8] and our papers [5]-[7]. However, up to now, only Proposition 1.3 in [6] gives conditions ensuring the existence of nonconstant (in particular, piecewise constant) lower and upper functions. The main

*Supported by the grant No.201/01/1451 of the Grant Agency of the Czech Republic and by the Council of Czech Government J14/98:153100011

†Supported by the grant No. 201/01/1199 of the Grant Agency of the Czech Republic

This paper is in final form and no version of it will be submitted for publications elsewhere.

EJQTDE, Proc. 7th Coll. QTDE, 2004 No. 19, p. 1 
goal of this paper is to find conditions for $f, \mathrm{~J}_{i}, \mathrm{M}_{i}$ giving piecewise linear lower and upper functions for (1.1)-(1.3). This together with the existence principles which we proved in [5]-[7] allows us to present new existence criteria.

Throughout the paper we keep the following notation and conventions: For $J \subset \mathbb{R}, \mathbb{C}(J)$ is the set of real valued functions which are continuous on $J, \mathbb{C}^{1}(J)$ is the set of functions having continuous first derivatives on $J, \mathbb{L}(J)$ is the set of functions Lebesgue integrable on $J$ and $\mathbb{L}_{\infty}(J)$ is the set of functions essentially bounded on $J$. For $u \in \mathbb{L}_{\infty}[0, T]$, we denote $\|u\|_{\infty}=\sup _{\operatorname{ess}_{t \in[0, T]}}|u(t)|$. Further, $\mathrm{D}=\left\{t_{1}, t_{2}, \ldots, t_{m}\right\}, t_{0}=0, t_{m+1}=T$ and $\mathbb{C}_{\mathrm{D}}^{1}[0, T]$ is the set of functions $u:[0, T] \mapsto \mathbb{R}$ of the form

$$
u(t)=\left\{\begin{array}{lc}
u_{[0]}(t) & \text { if } t \in\left[0, t_{1}\right], \\
u_{[1]}(t) & \text { if } t \in\left(t_{1}, t_{2}\right], \\
\cdots & \cdots \\
u_{[m]}(t) & \text { if } t \in\left(t_{m}, T\right]
\end{array}\right.
$$

where $u_{[i]} \in \mathbb{C}^{1}\left[t_{i}, t_{i+1}\right]$ for $i=0,1, \ldots, m$. Moreover, $\mathbb{A C}_{\mathrm{D}}^{1}[0, T]$ stands for the set of functions $u \in \mathbb{C}_{\mathrm{D}}^{1}[0, T]$ having first derivatives absolutely continuous on each subinterval $\left(t_{i}, t_{i+1}\right), i=0,1, \ldots, m$. For $u \in \mathbb{C}_{\mathrm{D}}^{1}[0, T]$ and $i=1,2, \ldots, m+1$ we define $u^{\prime}\left(t_{i}\right)=u^{\prime}\left(t_{i}-\right)=\lim _{t \rightarrow t_{i}-} u^{\prime}(t)$ and $u^{\prime}(0)=u^{\prime}(0+)=\lim _{t \rightarrow 0+} u^{\prime}(t)$.

1.1 Definition. A solution of the problem (1.1)-(1.3) is a function $u \in \mathbb{A}_{\mathrm{D}}^{1}[0, T]$ which satisfies the conditions (1.2) and (1.3) and for a.e. $t \in[0, T]$ fulfils the equation (1.1).

1.2 Definition. A function $\sigma_{1} \in \mathbb{A C}_{D}^{1}[0, T]$ is called a lower function of the problem (1.1)-(1.3) if

$$
\begin{aligned}
& \sigma_{1}^{\prime \prime}(t) \geq f\left(t, \sigma_{1}(t), \sigma_{1}^{\prime}(t)\right) \quad \text { for a.e. } t \in[0, T] \\
& \sigma_{1}\left(t_{i}+\right)=\mathrm{J}_{i}\left(\sigma_{1}\left(t_{i}\right)\right), \quad \sigma_{1}^{\prime}\left(t_{i}+\right) \geq \mathrm{M}_{i}\left(\sigma_{1}^{\prime}\left(t_{i}\right)\right), \quad i=1,2, \ldots, m, \\
& \sigma_{1}(0)=\sigma_{1}(T), \quad \sigma_{1}^{\prime}(0) \geq \sigma_{1}^{\prime}(T) .
\end{aligned}
$$

A function $\sigma_{2} \in \mathbb{A C}_{\mathrm{D}}^{1}[0, T]$ is an upper function of (1.1)-(1.3) if it satisfies

$$
\begin{aligned}
& \sigma_{2}^{\prime \prime}(t) \leq f\left(t, \sigma_{2}(t), \sigma_{2}^{\prime}(t)\right) \quad \text { for a.e. } t \in[0, T] \\
& \sigma_{2}\left(t_{i}+\right)=\mathrm{J}_{i}\left(\sigma_{2}\left(t_{i}\right)\right), \quad \sigma_{2}^{\prime}\left(t_{i}+\right) \leq \mathrm{M}_{i}\left(\sigma_{2}^{\prime}\left(t_{i}\right)\right), \quad i=1,2, \ldots, m, \\
& \sigma_{2}(0)=\sigma_{2}(T), \quad \sigma_{2}^{\prime}(0) \leq \sigma_{2}^{\prime}(T) .
\end{aligned}
$$

EJQTDE, Proc. 7th Coll. QTDE, 2004 No. 19, p. 2 


\section{Construction of nonconstant lower and upper functions}

2.1 Theorem. Assume (1.4) and

$$
\lim _{x \rightarrow \infty}\left(\mathrm{J}_{i}(x)-x\right)=c_{i} \in \mathbb{R} \text { for } i=1,2, \ldots, m .
$$

Denote

$$
c=-\frac{1}{T} \sum_{i=1}^{m} c_{i}
$$

and suppose that there are $A \in \mathbb{R}, \delta>0$ and $\nu \in\{1,2\}$ such that

$$
(-1)^{\nu} f(t, x, y) \geq 0 \text { for a.e. } t \in[0, T] \text { and all } x \geq A, y \in[c-\delta, c+\delta]
$$

$$
(-1)^{\nu}\left(M_{i}(y)-y\right) \geq 0 \text { for } y \in[c-\delta, c+\delta], i=1,2, \ldots, m .
$$

Then for each $\widetilde{A} \in[A, \infty)$ there exist $\widetilde{k} \in(c-\delta, c+\delta)$ and $\sigma_{\nu} \in \mathbb{A}_{\mathrm{D}}^{1}[0, T]$ such that $\sigma_{\nu}(t) \geq \widetilde{A}, \quad \sigma_{\nu}^{\prime}(t)=\widetilde{k}$ for $t \in[0, T]$ and, for $\nu=1(\nu=2), \sigma_{\nu}$ is a lower (upper) function of (1.1)-(1.3).

Proof. Let $\nu=2$ and $\widetilde{A} \in[A, \infty)$.

- Step 1. For $a, k \in \mathbb{R}, x \in \mathbb{R}$ and $i=1,2, \ldots, m$, define

$$
\varphi(t, a, k)= \begin{cases}a+k t & \text { if } t \in\left[0, t_{1}\right], \\ \mathrm{J}_{i}\left(\varphi\left(t_{i}, a, k\right)\right)+k\left(t-t_{i}\right) & \text { if } t \in\left(t_{i}, t_{i+1}\right], i=1,2, \ldots, m,\end{cases}
$$

and

$$
\varepsilon_{i}(x)=\mathrm{J}_{i}(x)-x-c_{i} .
$$

By virtue of (2.1), there are constants $A_{i}>\widetilde{A}, i=1,2, \ldots, m$, such that

$$
\left|\varepsilon_{i}(x)\right|<\frac{\delta T}{m} \quad \text { for all } x \geq A_{i} \text { and } i=1,2, \ldots, m \text {. }
$$

Furthermore, we have

$$
\varphi(t, a, k)=\left\{\begin{aligned}
a+k t \quad & \text { if } t \in\left[0, t_{1}\right] \\
a+k t+\sum_{j=1}^{i}\left(c_{j}+\varepsilon_{j}\left(\varphi\left(t_{j}, a, k\right)\right)\right) & \text { if } t \in\left(t_{i}, t_{i+1}\right], i=1,2, \ldots, m .
\end{aligned}\right.
$$

Put

$$
\widetilde{a}=\max _{j=1, \ldots, m} A_{j}+2\left(\sum_{j=1}^{m}\left|c_{j}\right|+\delta T\right)
$$

and suppose that $k \in[c-\delta, c+\delta]$. Then

EJQTDE, Proc. 7th Coll. QTDE, 2004 No. 19, p. 3 


$$
\varphi\left(t_{1}, \widetilde{a}, k\right)=\widetilde{a}+k t_{1} \geq A_{1}+\left(1-\frac{t_{1}}{T}\right) \sum_{j=1}^{m}\left|c_{j}\right| \geq A_{1}
$$

Furthermore, by (2.5), we have $\left|\varepsilon_{1}\left(\varphi\left(t_{1}, \widetilde{a}, k\right)\right)\right| \leq \frac{\delta T}{m}$. Consequently, in view of (2.6), we get

$$
\begin{aligned}
\varphi\left(t_{1}+, \widetilde{a}, k\right) & =\widetilde{a}+k t_{1}+c_{1}+\varepsilon_{1}\left(\varphi\left(t_{1}, \widetilde{a}, k\right)\right) \\
& \geq A_{2}+\left|c_{1}\right|+\delta T+\left(1-\frac{t_{1}}{T}\right) \sum_{j=1}^{m}\left|c_{j}\right|-\left|c_{1}\right|-\frac{\delta T}{m} \geq A_{2} .
\end{aligned}
$$

Now, let $1<i \leq m$ and let

$$
\varphi\left(t_{j}+, \widetilde{a}, k\right) \geq A_{j+1} \quad \text { and } \quad \varphi\left(t_{j}, \widetilde{a}, k\right) \geq A_{j}
$$

for each $j=1,2, \ldots, i-1$. Then, by (2.5), we have

$$
\left|\varepsilon_{j}\left(\varphi\left(t_{j}, \widetilde{a}, k\right)\right)\right| \leq \frac{\delta T}{m} \quad \text { for each } j=1,2, \ldots, i-1 .
$$

Hence, using (2.6) and (2.5), we get

$$
\varphi\left(t_{i}, \widetilde{a}, k\right) \geq A_{i}+\sum_{j=1}^{m}\left(1-\frac{t_{i}}{T}\right)\left|c_{j}\right|+\sum_{j=i}^{m}\left|c_{j}\right|+\left(1-\frac{i-1}{m}\right) \delta T \geq A_{i}
$$

and $\left|\varepsilon_{i}\left(\varphi\left(t_{i}, \widetilde{a}, k\right)\right)\right| \leq \frac{\delta T}{m}$. In view of $(2.6)$, we have

$$
\begin{aligned}
\varphi\left(t_{i}+, \widetilde{a}, k\right) & =\widetilde{a}+k t_{i}+\sum_{j=1}^{i}\left(c_{j}+\varepsilon_{j}\left(\varphi\left(t_{j}, \widetilde{a}, k\right)\right)\right) \\
& \geq A_{i+1}+\sum_{j=1}^{m}\left(1-\frac{t_{i}}{T}\right)\left|c_{j}\right|+\left(1-\frac{i}{m}\right) \delta T \geq A_{i+1}
\end{aligned}
$$

which means that $(2.7)$ is true for any $j \in\{1,2, \ldots, m\}$. Similarly we can show that $\varphi(T, \widetilde{a}, k) \geq A_{m}$. Thus

$$
\left\{\begin{aligned}
\varphi(t, \widetilde{a}, k) \geq \widetilde{A} & \text { and }\left|\varepsilon_{i}\left(\varphi\left(t_{i}, \widetilde{a}, k\right)\right)\right|<\frac{\delta T}{m} \\
& \text { for all } t \in[0, T], k \in[c-\delta, c+\delta] \text { and } i=1,2, \ldots, m .
\end{aligned}\right.
$$

- Step 2. We will prove that there is $\widetilde{k} \in(c-\delta, c+\delta)$ such that

$$
\varphi(0, \widetilde{a}, \widetilde{k})=\varphi(T, \widetilde{a}, \widetilde{k}) .
$$

EJQTDE, Proc. 7th Coll. QTDE, 2004 No. 19, p. 4 
By virtue of (2.6) and (2.8), $\sum_{i=1}^{m} \mid \varepsilon_{i}\left(\varphi\left(t_{i}, \widetilde{a}, k\right) \mid<\delta T\right.$ and

$$
\varphi(T, \widetilde{a}, k)-\varphi(0, \widetilde{a}, k)=T\left(k-c+\frac{1}{T} \sum_{i=1}^{m} \varepsilon_{i}\left(\varphi\left(t_{i}, \widetilde{a}, k\right)\right)\right)
$$

for each $k \in[c-\delta, c+\delta]$. In particular, $\varphi(T, \widetilde{a}, c-\delta)-\varphi(0, \widetilde{a}, c-\delta)<0$ and $\varphi(T, \widetilde{a}, c+\delta)-\varphi(0, \widetilde{a}, c+\delta)>0$. Since $\varphi$ is continuous, the existence of $\widetilde{k} \in(c-\delta, c+\delta)$ satisfying (2.9) follows.

- Step 3. Define $\sigma_{2}(t)=\varphi(t, \widetilde{a}, \widetilde{k})$ for $t \in[0, T]$. Then $\sigma_{2}^{\prime}(t)=\widetilde{k}$ for $t \in[0, T]$ and $\sigma_{2}\left(t_{i}+\right)=\mathrm{J}_{i}\left(\sigma_{2}\left(t_{i}\right)\right)$ for $i=1,2, \ldots, m$. By $(2.4)$ we have $\widetilde{k} \leq \mathrm{M}_{i}(\widetilde{k})$, i.e. $\sigma_{2}$ satisfies (1.9). Moreover, by (2.8) and (2.9), we have $\sigma_{2}(t) \geq \widetilde{A}$ on $[0, T]$ and $\sigma_{2}(0)=\sigma_{2}(T)$ and so (1.10) is true. Finally, by (2.3), $\sigma_{2}$ fulfils (1.8), i.e. $\sigma_{2}$ is an upper function for $(1.1)-(1.3)$.

The case $\nu=1$ can be treated analogously.

Theorem 2.1 gives piecewise linear lower and upper functions which are bounded below. Now we will show conditions guaranteeing the existence of lower or upper functions bounded above. This is the contents of the next theorem. Its proof is similar to that of Theorem 2.1.

2.2 Theorem. Assume (1.4). Further, let $d_{i} \in \mathbb{R}, i=1,2, \ldots, m, d, B \in \mathbb{R}, \eta>0$ and $\nu \in\{1,2\}$ be such that

$$
\begin{aligned}
& \lim _{x \rightarrow-\infty}\left(\mathrm{J}_{i}(x)-x\right)=d_{i} \in \mathbb{R} \text { for } i=1,2, \ldots, m, \quad d=-\frac{1}{T} \sum_{i=1}^{m} d_{i}, \\
& (-1)^{\nu} f(t, x, y) \geq 0 \text { for a.e. } t \in[0, T] \text { and all } x \leq B, y \in[d-\eta, d+\eta], \\
& (-1)^{\nu}\left(M_{i}(y)-y\right) \geq 0 \text { for } y \in[d-\eta, d+\eta], i=1,2, \ldots, m,
\end{aligned}
$$

Then for each $\widetilde{B} \leq B$ there exist $\widetilde{k} \in(d-\eta, d+\eta)$ and $\sigma_{\nu} \in \mathbb{A C}_{\mathrm{D}}^{1}[0, T]$ such that $\sigma_{\nu}(t) \leq \widetilde{B}, \quad \sigma_{\nu}^{\prime}(t)=\widetilde{k}$ for $t \in[0, T]$ and, for $\nu=1(\nu=2), \sigma_{\nu}$ is a lower (upper) function of (1.1)-(1.3).

2.3 Remark. Let (1.4) hold. Assume that $c_{i}, d_{i} \in \mathbb{R}$ and $A \in(0, \infty)$ are such that

$$
\begin{aligned}
& \mathrm{J}_{i}(x)=\left\{\begin{array}{l}
x+c_{i} \text { for } x \geq A \\
x+d_{i} \text { for } x \leq-A, i=1,2, \ldots, m,
\end{array}\right. \\
& \left\{\begin{array}{l}
f(t, x, c) \geq 0 \text { for a.e. } t \in[0, T] \text { and all } x \geq A, \\
f(t, x, d) \leq 0 \text { for a.e. } t \in[0, T] \text { and all } x \leq-A,
\end{array}\right.
\end{aligned}
$$

and

EJQTDE, Proc. 7th Coll. QTDE, 2004 No. 19, p. 5 


$$
\mathrm{M}_{i}(c) \geq c, \quad \mathrm{M}_{i}(d) \leq d, \quad i=1,2, \ldots, m .
$$

Let $\widetilde{A} \geq A$. Then, according to the proof of Theorem 2.1, we can see that

$$
\sigma_{2}(t)= \begin{cases}\widetilde{A}+\sum_{j=1}^{m}\left|c_{j}\right|+c t & \text { for } t \in\left[0, t_{1}\right), \\ \widetilde{A}+\sum_{j=1}^{m}\left|c_{j}\right|+c t+\sum_{j=1}^{i} c_{j} & \text { for } t \in\left(t_{i}, t_{i+1}\right], \quad i=1,2, \ldots, m\end{cases}
$$

and

$$
\sigma_{1}(t)= \begin{cases}-\widetilde{A}-\sum_{j=1}^{m}\left|d_{j}\right|+d t & \text { for } t \in\left[0, t_{1}\right), \\ -\widetilde{A}-\sum_{j=1}^{m}\left|d_{j}\right|+d t+\sum_{j=1}^{i} d_{j} & \text { for } t \in\left(t_{i}, t_{i+1}\right], \quad i=1,2, \ldots, m\end{cases}
$$

are respectively upper and lower functions of (1.1)-(1.3) satisfying

$$
\widetilde{A} \leq \sigma_{2}(t) \leq \widetilde{A}+2 \sum_{j=1}^{m}\left|c_{j}\right| \text { and } \quad-\widetilde{A}-2 \sum_{j=1}^{m}\left|d_{j}\right| \leq \sigma_{1}(t) \leq-\widetilde{A} \text { for } t \in[0, T] .
$$

If all inequalities in (2.10) and (2.11) are reversed, then $\sigma_{2}$ becomes a lower function and $\sigma_{1}$ an upper function.

\section{New existence criteria}

Our main results are Theorems 3.1 and 3.2 which provides new existence criteria for the problem (1.1)-(1.3).

3.1 Theorem. Let the assumptions of Theorem 2.1 be satisfied for $\nu=2$ and let the assumptions of Theorem 2.2 be satisfied for $\nu=1$. Assume that $\mathrm{J}_{i}$ are increasing on $\mathbb{R}$ and $\mathrm{M}_{i}$ are nondecreasing on $\mathbb{R}$ for $i=1,2, \ldots, m$. Finally, let for each compact interval $K \subset \mathbb{R}$ there exist $h_{K} \in \mathbb{L}[0, T]$ and $\omega_{K} \in \mathbb{C}([1, \infty))$ such that $h_{K} \geq 0$ on $[0, T], \omega_{K}>0$ on $[1, \infty), \int_{1}^{\infty} d s / \omega_{K}(s)=\infty$ and $|f(t, x, y)| \leq \omega_{K}(|y|)\left(|y|+h_{K}(t)\right)$ for a.e. $t \in[0, T]$ and all $x \in K,|y|>1$. Then the problem (1.1)-(1.3) has a solution.

Proof. By Theorem 2.1, for each $\widetilde{A} \geq A$, there is an upper function $\sigma_{2}$ of (1.1)-(1.3) such that $\sigma_{2} \geq \widetilde{A}$ on $[0, T]$. By Theorem 2.2, for each $\widetilde{B} \leq B$, there is a lower function $\sigma_{1}$ of (1.1)-(1.3) such that $\sigma_{1} \leq \widetilde{B}$ on $[0, T]$. Choose $\widetilde{A}, \widetilde{B}$ in such a way that $\widetilde{B} \leq \widetilde{A}$. Hence $\sigma_{1} \leq \sigma_{2}$ on $[0, T]$ and all the assumptions of [5, Theorem 3.1] are satisfied. Therefore (1.1)-(1.3) has a solution.

3.2 Theorem. Let the assumptions of Theorem 2.1 be satisfied for $\nu=1$ and let the assumptions of Theorem 2.2 be satisfied for $\nu=2$. Assume that $\mathrm{J}_{i}$ are increasing on $\mathbb{R}$ and $\mathrm{M}_{i}$ are nondecreasing on $\mathbb{R}$ for $i=1,2, \ldots, m$. Finally, let there exist $h \in \mathbb{L}[0, T]$ such that $|f(t, x, y)| \leq h(t)$ for a.e. $t \in[0, T]$ and all $x, y \in \mathbb{R}$. Then the problem (1.1)-(1.3) has a solution.

EJQTDE, Proc. 7th Coll. QTDE, 2004 No. 19, p. 6 
Proof. By Theorems 2.1 and 2.2 there are a lower function $\sigma_{1}$ and an upper function $\sigma_{2}$ of (1.1)-(1.3). The existence of a solution to (1.1)-(1.3) follows by [7, Theorem $3.1]$.

3.3 Example. Let $k \in \mathbb{N} \cup\{0\}, \gamma \in(0, \infty), p_{j} \in \mathbb{L}_{\infty}[0, T], j=0,1, \ldots, 2 k$, $p_{2 k+1} \in \mathbb{L}[0, T], p_{2 k+1} \geq \gamma$ a.e. on $[0, T], q_{1}, q_{2} \in \mathbb{L}_{\infty}[0, T], c_{i} \in(0, \infty), \alpha_{i} \in[0,1)$ and $\beta_{i} \in \mathbb{R}, i=1,2, \ldots, m$. Consider the problem (1.1)-(1.3), where

$f(t, x, y)=\sum_{j=0}^{2 k+1} p_{j}(t) x^{j}+q_{1}(t) y+q_{2}(t) y^{2}, \mathrm{~J}_{i}(x)=x+\frac{2 c_{i}}{\pi} \arctan x, \mathrm{M}_{i}(y)=\alpha_{i} y+\beta_{i}$

for a.e. $t \in[0, T]$, all $x, y \in \mathbb{R}$ and $i=1,2, \ldots, m$. Let $c$ be given by (2.2) and let $\beta_{i} \in\left(c\left(1-\alpha_{i}\right),-c\left(1-\alpha_{i}\right)\right), i=1,2, \ldots, m$. Then the conditions of Theorem 2.1 are satisfied for $\nu=2$ and the conditions of Theorem 2.2 are satisfied for $\nu=1$ and $d_{i}=-c_{i}, i=1,2, \ldots, m$. Since $c_{i}>0$ and $\alpha_{i} \geq 0$, the functions $\mathrm{J}_{i}$ are increasing and $\mathrm{M}_{i}$ are nondecreasing on $\mathbb{R}$ for $i=1,2, \ldots, m$. Choose an arbitrary compact interval $K \subset \mathbb{R}$ and denote $\varkappa_{1}=\max _{x \in K}\left(|x|^{2 k+1}\right)$ and $\varkappa_{2}=\sum_{j=1}^{2 k}\left(\left\|p_{j}\right\|_{\infty} \max _{x \in K}|x|^{j}\right)$. Then $|f(t, x, y)| \leq \omega(|y|)(|y|+h(t))$ for a.e. $t \in[0, T]$ and all $x \in K,|y|>1$, where $\omega(s)=1+\left\|q_{1}\right\|_{\infty}+\left\|q_{2}\right\|_{\infty} s$ and $h(t)=\varkappa_{1}\left|p_{2 k+1}(t)\right|+\varkappa_{2}$. Thus, the existence of a solution to (1.1)-(1.3) follows by means of Theorem 3.1.

3.4 Example. Let $\gamma \in(0, \infty), p \in \mathbb{L}_{\infty}[0, T], q_{1} \in \mathbb{L}[0, T], q_{2} \in \mathbb{L}_{\infty}[0, T], q_{1} \geq 0$, $q_{2} \geq 0$ and $q_{1}+q_{2} \geq \gamma$ a.e. on $[0, T], \varphi \in \mathbb{C}(\mathbb{R}), \lim _{|x| \rightarrow \infty} \varphi(x)=0$. Consider the problem (1.1)-(1.3), where

$$
f(t, x, y)=p(t) \varphi(x)+q_{1}(t) y+q_{2}(t) y^{2} \operatorname{sgn} y \text { for a.e. } t \in[0, T] \text { and all } x, y \in \mathbb{R}
$$

and $\mathrm{J}_{i}(x)$ and $\mathrm{M}_{i}(y), i=1,2, \ldots, m$, are given as in Example 3.3, but with $c_{i} \in$ $(-\pi / 2,0)$ and $\alpha_{i} \in(1, \infty), i=1,2, \ldots, m$. Let $c$ be given by $(2.2)$ and let $\beta_{i} \in$ $\left(c\left(1-\alpha_{i}\right), c\left(\alpha_{i}-1\right)\right)$ for $i=1,2, \ldots, m$. We have $c>0, M_{i}(c)>c, M_{i}(-c)<$ $-c, f(t, x, c)>0$ and $f(t, x,-c)<0$ for a.e. $t \in[0, T]$ and all $x \in \mathbb{R}$ with $|x|$ sufficiently large. Thus, the assumptions of Theorem 2.1 are satisfied for $\nu=2$ and the assumptions of Theorem 2.2 are satisfied for $\nu=1$ and $d_{i}=-c_{i}, i=1,2, \ldots, m$. Furthermore, $\mathrm{J}_{i}$ are increasing and $\mathrm{M}_{i}$ are nondecreasing on $\mathbb{R}$ for $i=1,2, \ldots, m$. Since $\varphi$ is bounded on $\mathbb{R}$, we can find $\omega \in \mathbb{C}([1, \infty))$ such that $\omega>0$ on $[1, \infty)$, $\int_{1}^{\infty} \mathrm{d} s / \omega(s)=\infty$ and $|f(t, x, y)| \leq \omega(|y|)|y|$ for a.e. $t \in[0, T]$ and all $x \in \mathbb{R},|y|>1$. Thus, by Theorem 3.1, the given problem has a solution.

3.5 Example. Let $\gamma \in(0, \infty), p \in \mathbb{L}_{\infty}[0, T], q \in \mathbb{L}[0, T], q \geq \gamma$ a.e. on $[0, T]$, $\varphi \in \mathbb{C}(\mathbb{R}), \lim _{|x| \rightarrow \infty} \varphi(x)=0$. Consider the problem (1.1)-(1.3), where

$$
f(t, x, y)=p(t) \varphi(x)+\frac{q(t) y}{1+y^{2}} \text { for a.e. } t \in[0, T] \text { and all } x, y \in \mathbb{R}
$$

EJQTDE, Proc. 7th Coll. QTDE, 2004 No. 19, p. 7 
and $\mathrm{J}_{i}(x)$ and $\mathrm{M}_{i}(y), i=1,2, \ldots, m$, are given as in Example 3.4, but with $c_{i} \in$ $(0, \infty)$. We can see that the assumptions of Theorem 2.1 are satisfied for $\nu=1$ and the assumptions of Theorem 2.2 are satisfied for $\nu=2$ and $d_{i}=-c_{i}, i=1,2, \ldots, m$. As in the previous examples, $\mathrm{J}_{i}$ are increasing and $\mathrm{M}_{i}$ are nondecreasing on $\mathbb{R}$ for $i=1,2, \ldots, m$. Moreover, since the functions $\varphi$ and $\frac{y}{y^{2}+1}$ are bounded on $\mathbb{R}$, we can find $h \in \mathbb{L}[0, T]$ such that $|f(t, x, y)| \leq h(t)$ for a.e. $t \in[0, T]$ and all $x, y \in \mathbb{R}$. Thus, by Theorem 3.2, our problem has a solution.

\section{References}

[1] D. Bainov and P. Simeonov, Impulsive Differential Equations: Periodic Solutions and Applications. Longman Sci. Tech., Harlow, 1993.

[2] Dong Yujun. Periodic solutions for second order impulsive differential systems. Nonlinear Anal. 27 (1996), 811-820.

[3] Hu Shouchuan and V. Laksmikantham. Periodic boundary value problems for second order impulsive differential systems. Nonlinear Anal. 13 (1989), 75-85.

[4] E. Liz And J. J. Nieto. Periodic solutions of discontinuous impulsive differential systems. J. Math. Anal. Appl. 161 (1991), 388-394.

[5] I. RachŮnková And M. TvRDÝ. Periodic boundary value problems for nonlinear second order differential equations with impulses - Part I. Math. Inst. Acad. Sci Czech Rep., Preprint 148/2002, available on \http://www.math.cas.cz/ tvrdy/i2.ps, to appear as "Nonmonotone impulse effects in second order periodic boundary value problems" in Abstr. Anal. Appl..

[6] I. RachŮnková And M. TVRDÝ. Periodic boundary value problems for nonlinear second order differential equations with impulses - Part II. Math. Inst. Acad. Sci Czech Rep., Preprint 151/2002, available on \http: //www.math.cas.cz/ tvrdy/i3.ps.

[7] I. Rachůnková And M. TvRdÝ. Periodic boundary value problems for nonlinear second order differential equations with impulses - Part III. Math. Inst. Acad. Sci Czech Rep., Preprint 153/2003, available on \http: //www.math.cas.cz/ tvrdy/i4.ps.

[8] Zhang Zhitao. Existence of solutions for second order impulsive differential equations. Appl.Math., Ser.B (Engl.Ed.) 12, (1997), 307-320.

Irena Rachůnková, Department of Mathematics, Palacký University, 77900 OLOMOUC, Tomkova 40, Czech Republic (e-mail: rachunko@risc.upol.cz)

Milan Tvrdý, Mathematical Institute, Academy of Sciences of the Czech Republic, 11567 PRAHA 1, Žitná 25, Czech Republic (e-mail: tvrdy@math.cas.cz)

(Received September 30, 2003)

EJQTDE, Proc. 7th Coll. QTDE, 2004 No. 19, p. 8 